\title{
Monitoring of post-transplant MLL-PTD as minimal residual disease can predict relapse after allogeneic HSCT in patients with acute myeloid leukemia and myelodysplastic syndrome
}

Jun Kong ${ }^{1 \dagger}$, Meng-Ge Gao ${ }^{1+}$, Ya-Zhen Qin ${ }^{1}$, Yu Wang ${ }^{1}$, Chen-Hua Yan ${ }^{1,2}$, Yu-Qian Sun ${ }^{1}$, Ying-Jun Chang ${ }^{1,2,3}$, Lan-Ping X ${ }^{1,2}$, Xiao-Hui Zhang ${ }^{1}$, Kai-Yan Liu' ${ }^{1}$, Xiao-Jun Huang ${ }^{1,2,3,4}$ and Xiao-Su Zhao ${ }^{1,2,3^{*}}$

\begin{abstract}
Background: The mixed-lineage leukemia (MLL) gene is located on chromosome 11q23. The MLL gene can be rearranged to generate partial tandem duplications (MLL-PTD), which occurs in about 5-10\% of acute myeloid leukemia (AML) with a normal karyotype and in 5-6\% of myelodysplastic syndrome (MDS) patients. Allogeneic hematopoietic stem cell transplantation (allo-HSCT) is currently one of the curative therapies available for AML and MDS with excess blasts (MDS-EB). However, how the prognosis of patients with high levels of MLL-PTD after allo-HSCT, and whether MLL-PTD could be used as a reliable indicator for minimal residual disease (MRD) monitoring in transplant patients remains unknown. Our study purposed to analyze the dynamic changes of MLL-PTD peri-transplantation and the best threshold for predicting relapse after transplantation.
\end{abstract}

Methods: We retrospectively collected the clinical data of 48 patients with MLL-PTD AML or MDS-EB who underwent allo-HSCT in Peking University People's Hospital. The MLL-PTD was examined by real-time quantitative polymerase chain reaction (RQ-PCR) at the diagnosis, before transplantation and the fixed time points after transplantation. Detectable MLL-PTD/ABL $>0.08 \%$ was defined as MLL-PTD positive in this study.

Results: The 48 patients included 33 AML patients and 15 MDS-EB patients. The median follow-up time was 26(0.756) months after HSCT. In AML patients, 7 patients (21.2\%) died of treatment-related mortality (TRM), 6 patients (18.2\%) underwent hematological relapse and died ultimately. Of the 15 patients with MDS-EB, 2 patients (13.3\%) died of infection. The 3-year cumulative incidence of relapse (CIR), overall survival (OS), disease-free survival (DFS) and TRM were $13.7 \pm 5.2,67.8 \pm 6.9,68.1 \pm 6.8$ and $20.3 \% \pm 6.1 \%$, respectively. ROC curve showed that post-transplant MLLPTD $\geq 1.0 \%$ was the optimal cut-off value for predicting hematological relapse after allo-HSCT. There was statistical difference between post-transplant MLL-PTD $\geq 1.0 \%$ and MLL-PTD $<1.0 \%$ groups (3-year CIR: $75 \% \pm 15.3 \%$ vs. 0\%,

\footnotetext{
*Correspondence: zhao.xiaosu@outlook.com

†Jun Kong and Meng-Ge Gao contributed equally to this work.

1 Beijing Key Laboratory of Hematopoietic Stem Cell Transplantation

Peking University People's Hospital, Peking University Institute

of Hematology, National Clinical Research Center for Hematologic

Disease, No 11 Xizhimen South Street, Beijing 100044, China

Full list of author information is available at the end of the article
}

(c) The Author(s) 2021. Open Access This article is licensed under a Creative Commons Attribution 4.0 International License, which permits use, sharing, adaptation, distribution and reproduction in any medium or format, as long as you give appropriate credit to the original author(s) and the source, provide a link to the Creative Commons licence, and indicate if changes were made. The images or other third party material in this article are included in the article's Creative Commons licence, unless indicated otherwise in a credit line to the material. If material is not included in the article's Creative Commons licence and your intended use is not permitted by statutory regulation or exceeds the permitted use, you will need to obtain permission directly from the copyright holder. To view a copy of this licence, visit http://creativecommons.org/licenses/by/4.0/. The Creative Commons Public Domain Dedication waiver (http://creativeco mmons.org/publicdomain/zero/1.0/) applies to the data made available in this article, unless otherwise stated in a credit line to the data. 
$P<0.001 ; 3$-year OS: $25.0 \pm 15.3 \%$ vs. $80.7 \% \pm 6.6 \%, P<0.001$; 3-year DFS: $25.0 \pm 15.3 \%$ vs. $80.7 \pm 6.6 \%, P<0.001$; 3-year TRM: 0 vs. $19.3 \pm 6.6 \%, P=0.277$ ). However, whether MLL-PTD $\geq 1 \%$ or MLL-PTD $<1 \%$ before transplantation has no significant difference on the prognosis.

Conclusions: Our study indicated that MLL-PTD had a certain stability and could effectively reflect the change of tumor burden. The expression level of MLL-PTD after transplantation can serve as an effective indicator for predicting relapse.

Keywords: MLL-PTD, Minimal residual disease, Allogeneic hematopoietic stem cell transplantation, Relapse

\section{Background}

Acute myeloid leukemia (AML) is a highly malignant hematopoietic system disease and myelodysplastic syndrome (MDS) is a type of heterogeneous myeloid malignancies and frequently progress to AML [1-4]. In previous studies, molecular genetic aberrations have become important approaches for minimal residual disease (MRD) detection for AML and MDS. Especially, the polymerase chain reaction (PCR)-based gene detection has been proven to be an effective MRD monitoring method for AML patients [5-7]. However, more than half of AML cases still lack effective specific MRD molecular markers [5].

The mixed-lineage leukemia $(M L L)$ gene, also named lysine (K)-specific methyltransferase 2A (KMT2A), is located on chromosome 11q23. Genetic alterations of the MLL gene are usually associated with the development of acute leukemia [8]. The MLL gene may be rearranged to generate partial tandem duplications ( $M L L-\mathrm{PTD})$, which usually spans exons 2 to 6,2 to 7 , and 2 to 8 , or exons $3-9$, exons $3-10$, exons $3-11$, or exons $3-10$ and exons 3-11 at the molecular level [8-11]. MLL-PTD has been detected in approximately $5-10 \%$ of AML and $5-6 \%$ of MDS patients [12-14]. Low level of $M L L-P T D(<0.08 \%)$ may also be present in the blood and bone marrow of healthy individuals [5]. Previous reports support that polymerase chain reaction (PCR)-based $M L L$-PTD is a reliable MRD marker and is associated with poor prognosis [5, 12-15]. For chemotherapy patients, a higher $M L L-$ PTD level at initial diagnosis predicts a lower incidence of chemotherapy complete remission (CR) and a lower survival rate [13]. The dynamic changes of chemotherapy patients also show that $M L L$-PTD levels within the first 6 months after the start of therapy are useful for early risk assessment of AML patients, and that a reduction of $M L L$-PTD level $\geq 2 \log$ is a good prognostic factor for overall survival [5]. Furthermore, compared with healthy donors, $M L L$-PTD level have no difference from that of non-transplanted patients in continuous $C R$, while was significantly higher than that of transplanted patients in continuous CR [15]. Taken together, these findings support that $M L L$-PTD is a specific clinical prognostic marker in the initial diagnosis and chemotherapy for
AML patients. However, there are few reports on the dynamics of $M L L-P T D$ peri-transplantation, especially after transplantation. Thus, whether $M L L$-PTD could be used as a stable and reliable MRD marker in the process of transplantation and whether there is an optimal value of $M L L-P T D$ to predict relapse after transplantation will be explored for the first time in our study.

In this study, we investigated a consecutive cohort of 33 AML and 15 MDS patients with $M L L$-PTD who received allo-HSCT at our institute. Most $M L L$-PTD MDS cases are classified as MDS with excess blasts (MDS-EB) [16]. Our study purposed to analyze the dynamic changes of $M L L$-PTD peri-transplantation and the best threshold for predicting relapse after transplantation.

\section{Methods \\ Patients}

The consecutive patients diagnosed with $M L L$-PTD expression $>0.08 \%$ AML or MDS undergoing allo-HSCT between January 2015 and March 2019 at the Peking University People's Hospital, Institute of Hematology were enrolled in this study. The patients' data were updated until September 30, 2020. The institutional review board at the hospital approved the protocol, and all patients or their guardians signed consent forms approved by the institutional review board.

\section{Transplantation protocol}

All the patients in this study received myeloablative conditioning regimens. Haploidentical HSCT (haplo-HSCT) and matched sibling donor transplantation (MSDT) were performed according to protocols reported previously by our institute $[17,18]$. The conditioning regimen for MSDT patients is: Cytarabine (Ara-C) $2 \mathrm{~g} / \mathrm{m}^{2} / \mathrm{d}$ i.v. for 1 day, cyclophosphamide (CTX) $1.8 \mathrm{~g} / \mathrm{m}^{2} / \mathrm{d}$ for 2 days, busulfan (BU) $0.8 \mathrm{mg} / \mathrm{kg}$ i.v., q.i.d. for 3 days, and nitrosourea (Simustine, MeCCNU) $250 \mathrm{mg} / \mathrm{kg}$ for 1 day. The conditioning regimen for haplo-HSCT patients is: Ara-C $4 \mathrm{~g} / \mathrm{m}^{2} / \mathrm{d}$ i.v. for 2 days, CTX $1.8 \mathrm{~g} / \mathrm{m}^{2} / \mathrm{d}$ for 2 days, BU $0.8 \mathrm{mg} / \mathrm{kg}$ i.v., q.i.d. for 3 days, and MeCCNU $250 \mathrm{mg} /$ $\mathrm{kg}$ for 1 day, and thymoglobulin (ATG, Sang Stat, Lyon, France) $2.5 \mathrm{mg} / \mathrm{kg} / \mathrm{d}$ i.v. for 4 days. 


\section{Donor Lymphocyte Infusion (DLI)}

Prophylactic DLI was administered for patients in relapse or no remission (NR) state before transplantation. The indications for DLI included hematological leukemia relapse, receiving chemotherapy followed by DLI, or positive MRD detection as previously described [19].

\section{Detection of MRD}

In this study, MRD was evaluated by Flow Cytometry (FCM) [20], the expression level of WT1 and MLL-PTD determined by RQ-PCR. The pre-transplant FCM, $M L L-$ PTD and WT1 were performed using bone marrow (BM) samples within a month before the transplant as a routine. The post-transplant scheduled time points were +1 , $+2,+3,+4.5,+6,+9$, and +12 months post-HSCT and every 6 months thereafter.

The patients were analyzed for the presence of MLLPTD at the MLL gene locus, as described previously [13, 15]. Briefly, $M L L$ primers and hybridization probes were placed in exons 8-10 and 3 of the $M L L$ gene, allowing for detection of $M L L-\mathrm{PTD}$ with exon 8/exon 3 fusion, exon 9/exon 3 fusion, or exon 10/exon 3 fusion. The transcript level was calculated as target transcript copies/ABL copies in percentages. Detectable $M L L$-PTD $/ A B L>0.08 \%$ was defined as $M L L$-PTD positive [13]. The $W T 1$ was detected as described previously and a WT1 transcript level less than $0.60 \%$ was defined as negative [21].

\section{Definitions and assessments}

The day of neutrophil engraftment was defined as the first day of 3 consecutive post-transplantation days on which the absolute neutrophil count (ANC) exceeded $500 / \mu \mathrm{L}$. Patients who survived at least 28 days were considered to have had successful engraftment. The criteria for grading acute graft versus host disease (aGVHD) have been previously published [22, 23]. CR was defined as hematological CR that is, $<5 \%$ BM blasts, the absence of blasts in peripheral blood, the absence of extramedullary disease, an $\mathrm{ANC}>1.0 \times 10^{9} / \mathrm{L}$, and a platelet count $>100 \times 10^{9} / \mathrm{L}$ with no red cell transfusions. Hematological relapse was defined by morphologic evidence of disease in the peripheral blood, marrow, or extramedullary sites.

\section{Statistical analysis}

The primary study end point was the cumulative incidence of relapse (CIR). The secondary end points were the OS, disease-free survival (DFS) and treatment-related mortality (TRM). CIR, OS, DFS and TRM were defined as previously described [24]. Summary statistics, such as proportions, medians and ranges, were used to describe the patient characteristics and outcomes. The associations between $M L L$-PTD expression and post-transplantation outcomes were analyzed by the Kaplan-Meier method. Differences in CIR, DFS, OS and TRM between groups were calculated using the log-rank test. A two-sided $P$ value of 0.05 was considered statistically significant. The independence of categorical parameters was calculated using the chi-square test or Fisher exact test, and the distribution of continuous variables was calculated using the Mann-Whitney U-test. All statistical analyses were performed using SPSS 23.0 (Chicago, IL, USA).

\section{Results}

\section{Patients characteristics}

A total of 33 AML patients included 13 males and 20 females, with a median age of 42 years (10-57 years) and 15 MDS-EB patients included 11 males and 4 females, with a median age of 51 years (4-60years). The median follow-up time was 26 (0.7-56) months after HSCT. Patient characteristics are shown in Table 1. Of these 33 AML patients, 31 patients had gotten CR after chemotherapy, and 2 patients had gotten NR after 3 courses of chemotherapy. And 5 MDS-EB patients receiving chemotherapy including decitabine had gotten CR pretransplantation. All patients had neutrophil engraftment, and 39 patients had platelet engraftment. Of the 33 patients with AML, 7 patients (21.2\%) died of TRM and 6 patients (18.2\%) underwent hematological relapse who died ultimately. The median hematological relapse time was 4.8 months (range 4-9 months) after HSCT in 6 relapsed patients. Of the 15 patients with MDS-EB, 2 patients (13.3\%) died of infection. In addition, all enrolled patients had a 3-year CIR of $13.7 \% \pm 5.2 \%$, 3-year OS of $67.8 \% \pm 6.9 \%$, 3-year DFS of $68.1 \% \pm 6.8 \%$ and 3-year TRM of $20.3 \% \pm 6.1 \%$ (Fig. 1).

\section{Dynamic changes of MLL-PTD before and after transplantation}

Observing the changes in the expression level of $M L L$ PTD at different time points peri-transplantation is helpful to analyze the stability of $M L L-P T D$ as an MRD indicator in the transplantation system. Our results showed that the $M L L$-PTD level before transplantation was significantly lower than that at the initial diagnosis, but there were still 37 cases were $M L L$-PTD positive before transplantation, and 33 of 37 cases became negative within post-transplant 1 month. However, during our follow-up period, 25 cases eventually occurred posttransplant $M L L$-PTD positive. The median $M L L$-PTD level in all enrolled patients was decreased by around 35 folds after transplantation compared with that of pre-transplant CR status and was similar to the healthy controls (Table 2). Furthermore, among the 6 relapsed 
Table 1 Characteristics of acute myeloid leukemia and myelodysplastic syndrome patients

\begin{tabular}{|c|c|c|}
\hline Characteristic & AML $N=33$ & MDS-EB $1 / 2 N=15$ \\
\hline Median age at allo-HCT, years (range) & $42(10-57)$ & $51(4-60)$ \\
\hline \multicolumn{3}{|l|}{ Gender, n (\%) } \\
\hline Male & $13(39.4 \%)$ & $11(73.3 \%)$ \\
\hline Female & $20(60.6 \%)$ & $4(26.7 \%)$ \\
\hline Chromosome normal, n (\%) & $23(69.7 \%)$ & $9(60.0 \%)$ \\
\hline \multicolumn{3}{|l|}{ FLT3-ITD mutation, n (\%) } \\
\hline Yes & $10(30.3 \%)$ & 0 \\
\hline No & $23(69.7 \%)$ & $15(100 \%)$ \\
\hline NPM1 mutation, n (\%) & 0 & 0 \\
\hline \multicolumn{3}{|l|}{ Risk category } \\
\hline Favorable & 0 & 0 \\
\hline Intermediate & 33 & 15 \\
\hline Adverse & 0 & 0 \\
\hline Median WT1 expression level at initial diagnosis & $25.25(0.23-83.20)$ & $18.80(1.40-53.50)$ \\
\hline No remission before transplant, $\mathrm{n}(\%)$ & $2(6.1 \%)$ & $1(6.7 \%)$ \\
\hline \multicolumn{3}{|l|}{ Donor type, n (\%) } \\
\hline HLA-matched sibling & $7(21.2 \%)$ & $5(33.3 \%)$ \\
\hline Haploidentical & $26(78.8 \%)$ & 10(66.7\%) \\
\hline \multicolumn{3}{|l|}{ ABO blood type match, $\mathrm{n}(\%)$} \\
\hline Compatible & $17(51.5 \%)$ & $7(46.7 \%)$ \\
\hline Incompatible & $16(48.5 \%)$ & $8(53.3 \%)$ \\
\hline \multicolumn{3}{|l|}{ Conditioning regimen, $\mathrm{n}(\%)$} \\
\hline Chemotherapy based & $33(100 \%)$ & $15(100 \%)$ \\
\hline TBI based & 0 & 0 \\
\hline \multicolumn{3}{|l|}{ Cell compositions in allografts } \\
\hline Median MNC, $\times 10^{8} / \mathrm{kg}$ (range) & $7.82(6.04-10.86)$ & $8.54(6.10-10.86)$ \\
\hline Median CD34+ count, $\times 10^{6} / \mathrm{kg}$ (range) & $2.32(0.27-6.67)$ & $1.89(0.84-5.34)$ \\
\hline Granulocyte engraftment time, day (range) & $13(8-25)$ & $13(11-19)$ \\
\hline Platelet engraftment time, day (range) & $14(10-74)$ & $13(10-53)$ \\
\hline II-IV’aGVHD & $8(24.2 \%)$ & $1(6.7 \%)$ \\
\hline $\mathrm{aGVHD}$ & $18(54.5 \%)$ & $4(26.7 \%)$ \\
\hline cGVHD & $5(15.2 \%)$ & $5(33.3 \%)$ \\
\hline \multicolumn{3}{|l|}{ DLI after transplant, n (\%) } \\
\hline For relapse prevention & $2(6.1 \%)$ & 0 \\
\hline For intervention & $4(12.1 \%)$ & $2(13.3 \%)$ \\
\hline \multicolumn{3}{|l|}{ Prognosis, n (\%) } \\
\hline Relapse & $6(18.2 \%)$ & 0 \\
\hline Treatment-related death & $7(21.2 \%)$ & $2(13.3 \%)$ \\
\hline Relapse death & $6(18.2 \%)$ & 0 \\
\hline
\end{tabular}

$A M L$ acute myeloid leukemia, $M D S$ myelodysplastic syndrome, $H L A$ human leukocyte antigen, $T B I$ total body irradiation, $M N C$ mononuclear cell, $a G V H D$ acute graft versus host disease, $C G V H D$ chronic graft versus host disease, $D L I$ donor lymphocyte infusion

patients after transplantation, 3 of them maintained $M L L$-PTD at the healthy level $(<0.08 \%)$ within a month after transplantation. But before relapse, the $M L L-\mathrm{PTD}$ level of these 3 patients gradually increased $(>0.08 \%)$ and reached the highest level at the time of relapse. The $M L L$-PTD level of the other 3 relapsed patients continuously remained $>0.08 \%$ after transplantation, and the
$M L L$-PTD levels of these 3 patients suddenly increased by hundreds of times before relapse.

\section{The effect of MLL-PTD level before and after transplantation on prognosis}

Having analyzed the dynamic changes above which peri-transplant $M L L-$ PTD can stably reflect the disease 

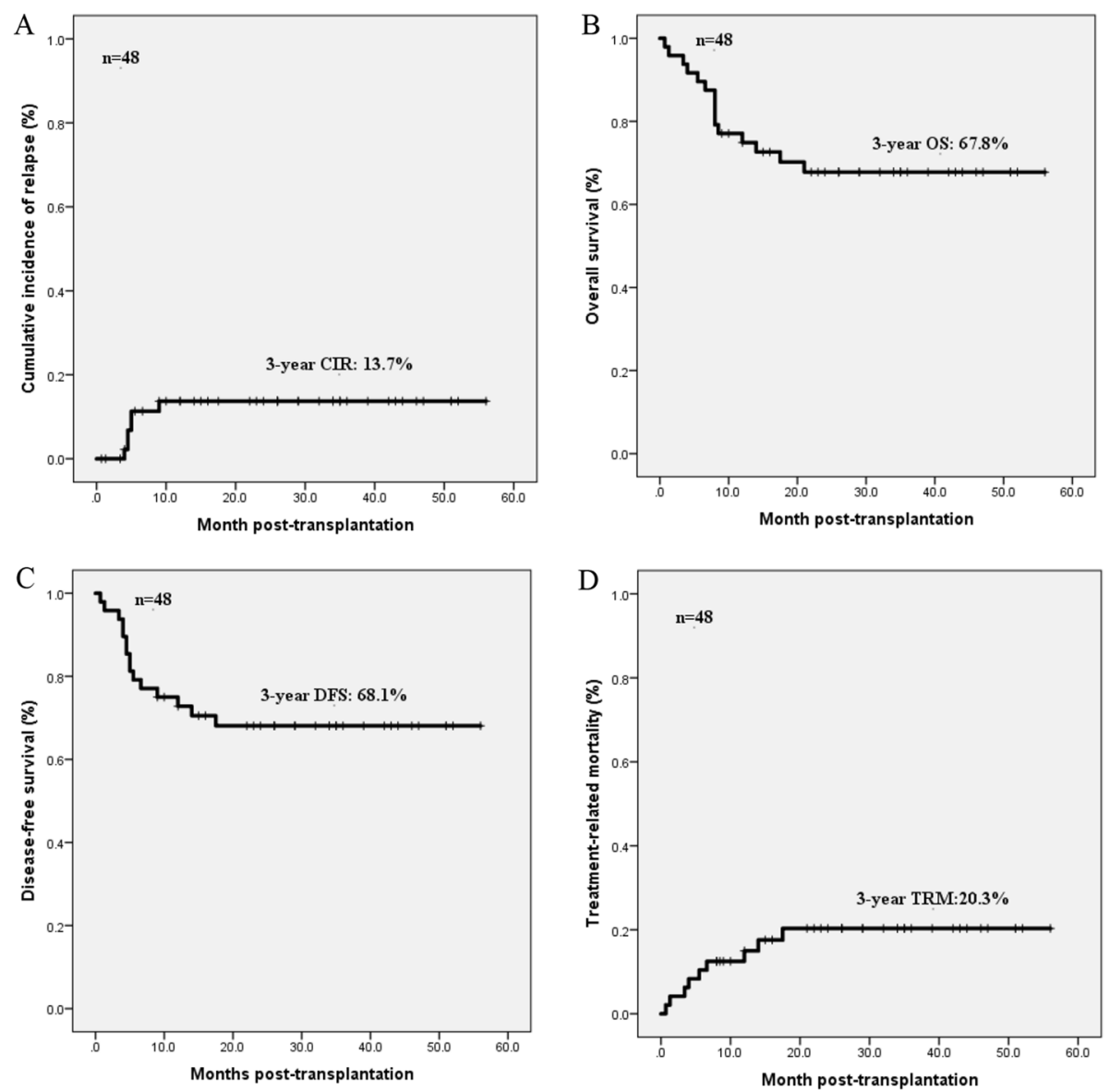

Fig. 1 Cumulative incidence of relapse, overall survival, disease-free survival and treatment-related mortality of 48 MLL-PTD patients after allo-HSCT

Table 2 Comparison of MLL-PTD and WT1 at the initial diagnosed and peri-transplant patients

\begin{tabular}{|c|c|c|c|c|c|c|}
\hline & $\begin{array}{l}\text { MLL-PTD }>0.08 \%(\mathrm{n} / \\
\text { total tests, positive } \\
\text { rate) }\end{array}$ & $\begin{array}{l}\text { Median level of MLL- } \\
\text { PTD }>0.08 \% \text { (range, } \\
\% \text { ) }\end{array}$ & $\begin{array}{l}\text { Median level of } \\
\text { MLL-PTD (range, } \\
\% \text { ) }\end{array}$ & $\begin{array}{l}\text { WT1 }>0.6 \% \\
\text { (n/total } \\
\text { tests) }\end{array}$ & $\begin{array}{l}\text { Median level of } \\
\text { WT1 }>0.6 \% \text { (range, } \\
\% \text { ) }\end{array}$ & $\begin{array}{l}P \text { value (MLL- } \\
\text { PTD+ vs. } \\
\text { WT1+) }\end{array}$ \\
\hline The initial diagnosis & 48/48(100\%) & $30.30(1.20-631.00)$ & $30.30(1.20-631.00)$ & 44/47(93.6\%) & $26.20(0.82-83.20)$ & 0.233 \\
\hline Pre-transplantation & $37 / 48(68.8 \%)$ & $6.10(0.10-414.10)$ & $1.70(0.017-414.10)$ & 28/47(59.6\%) & $6.20(0.88-53.50)$ & 0.351 \\
\hline \multicolumn{7}{|l|}{ Post-transplantation } \\
\hline+1 month & $8 / 43(18.6 \%)$ & $0.115(0.083-0.73)$ & $0.046(0.01-0.73)$ & $1 / 46(2.2 \%)$ & 0.82 & 0.027 \\
\hline+2 month & $12 / 44(27.3 \%)$ & $0.21(0.09-0.82)$ & $0.047(0-0.82)$ & $3 / 45(6.7 \%)$ & $0.86(0.74-2.4)$ & 0.009 \\
\hline+3 month $^{\mathrm{a}}$ & 13/45(28.9\%) & $0.28(0.086-104.70)$ & $0.05(0-104.70)$ & $6 / 46(13.0 \%)$ & $1.50(0.75-32.70)$ & 0.063 \\
\hline+4.5 month $^{\mathrm{a}}$ & 8/38(21.1\%) & $1.30(0.082-55.30)$ & $0.0515(0-55.30)$ & 9/39(23.1\%) & $3.90(0.81-44.10)$ & 0.524 \\
\hline+6 month $^{\mathrm{a}}$ & 11/39(28.2\%) & $1.40(0.096-101.30)$ & $0.053(0.015-101.30)$ & 12/39(30.8\%) & $1.30(0.60-80.90)$ & 0.500 \\
\hline+9 month $^{\mathrm{a}}$ & $5 / 27(18.5 \%)$ & $0.09(0.08-0.11)$ & $0.0445(0-1.00)$ & 3/34(8.8\%) & $0.71(0.63-0.74)$ & 0.231 \\
\hline+12 month & $1 / 30(3.3 \%)$ & 0.45 & $0.049(0-0.45)$ & $5 / 32(15.6 \%)$ & $0.88(0.72-1.00)$ & 0.113 \\
\hline
\end{tabular}

${ }^{a}$ Patients underwent hematological relapse at that time point 
A

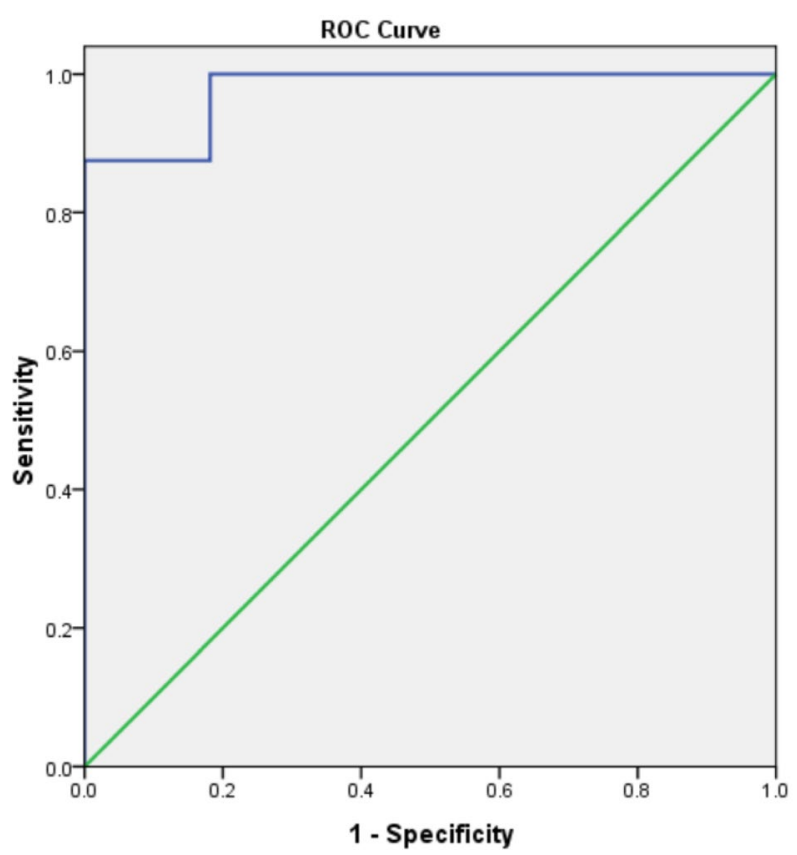

B

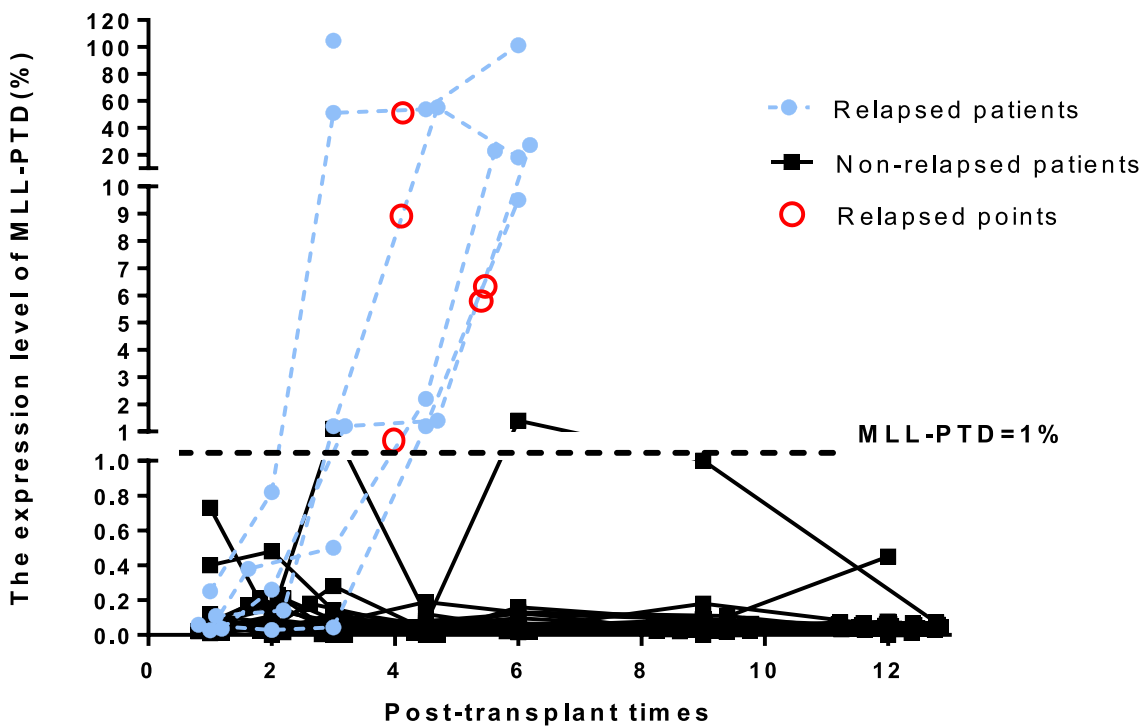

Fig. 2 The curve of MLL-PTD expression levels post-transplantation. A Receiver operating characteristic (ROC) curve of MLL-PTD expression post-transplantation ( $A \cup C=0.977, P<0.001$ ). $\mathbf{B}$ The level changes of post-transplant MLL-PTD

state we next studied the optimal threshold of posttransplant $M L L$-PTD for relapse. Our previous study shows that patients with $M L L-\mathrm{PTD} / A B L \geq 1 \%$ based on initial diagnosis have a poor clinical prognosis [13]. In order to explore whether $M L L-\mathrm{PTD}$ could be used as a MRD marker after transplantation, we performed a receiver operating characteristic (ROC) with the highest expression level of post-transplant $M L L$-PTD before hematological relapse in all patients to determine the optimal cut-off value to predict relapse. The area under the ROC curve value was $0.977(P<0.001$, Fig. 2A). The optimal cut-off value was $M L L-\mathrm{PTD} / A B L=1.0 \%$. And as shown in Fig. 2B, most post-transplant patients with $M L L$-PTD maintained a low level of expression, only 8 patients had $M L L-\mathrm{PTD} \geq 1 \%$, and 6 of the 8 patients eventually relapsed, which also implied the importance of $M L L-\mathrm{PTD} \geq 1 \%$ in predicting relapse after transplantation. Based on the optimal cut-off value, we 

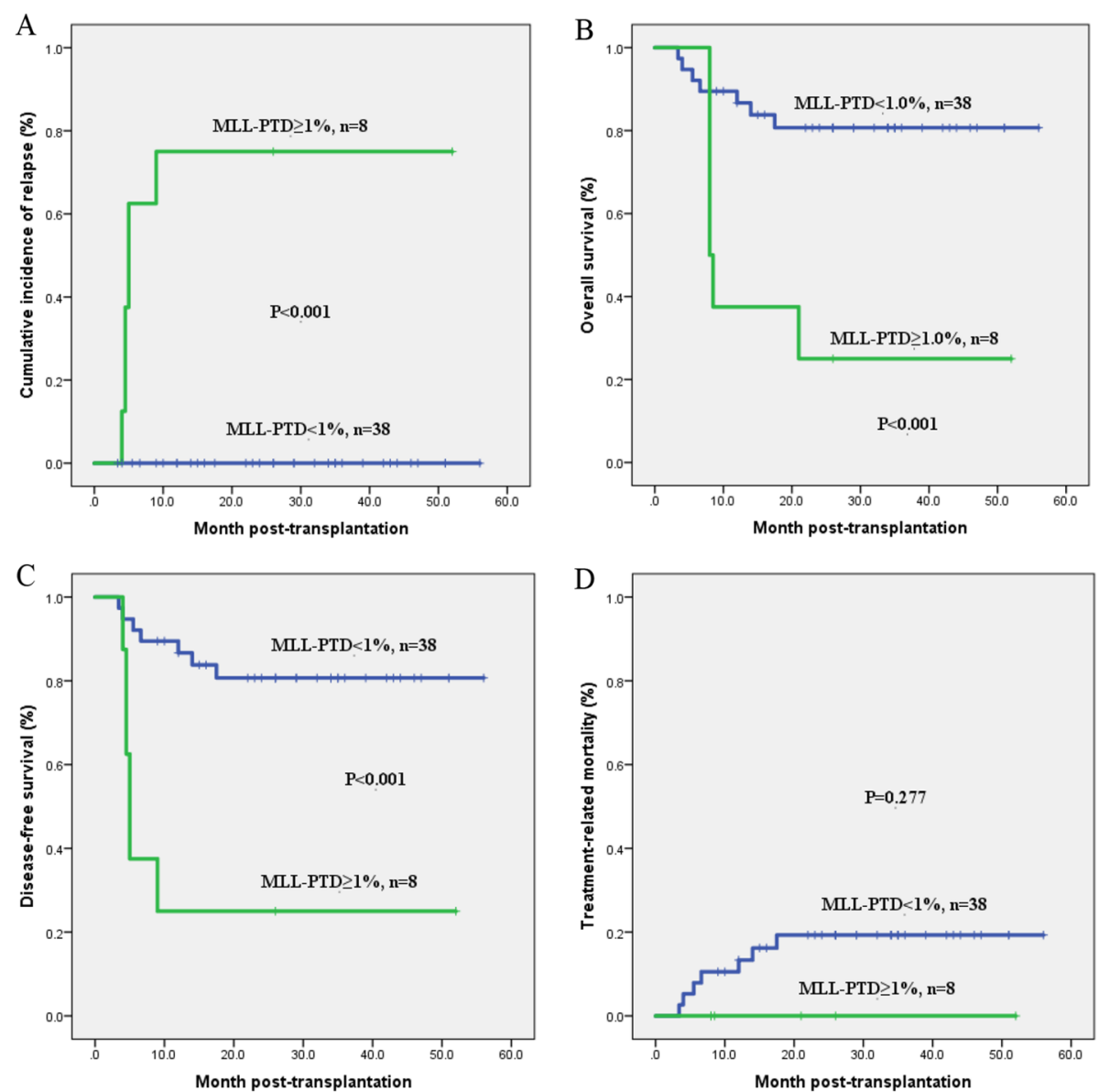

Fig. 3 Kaplan-Meier survival curves analysis of patients between MLL-PTD $<1 \%$ and MLL-PTD $\geq 1 \%$ after transplantation

divided the post-transplant patients into two groups of $M L L-\mathrm{PTD} / A B L<1 \%$ and $M L L-\mathrm{PTD} / A B L \geq 1 \%$ to analyzed the prognostic difference. Our study found that the group of $M L L$-PTD $/ A B L \geq 1.0 \%$ had higher 3-year CIR ( $75 \pm 15.3 \%$ vs. $0 \%, P<0.001$, Fig. $3 \mathrm{~A})$, and lower 3 -year OS $(25.0 \pm 15.3 \%$ vs. $80.7 \% \pm 6.6 \%, P<0.001$, Fig. 3B) and 3 -year DFS (25.0 $\pm 15.3 \%$ vs. $80.7 \pm 6.6 \%$, $P<0.001$, Fig. 3C) compared with that of group of $M L L-$ $\mathrm{PTD} / A B L<1 \%$. However, there was no statistical difference between the two groups in TRM $(P>0.05$, Fig. 3D).

Both at the initial diagnosis and post-transplantation, it was analyzed that $M L L-\mathrm{PTD}=1 \%$ was the optimal cut-off value, which implied that $M L L-\mathrm{PTD} / \mathrm{ABL}=1 \%$ was of important value in predicting prognosis. Therefore, we further analyzed whether $M L L-\mathrm{PTD} / A B L \geq 1 \%$ before transplantation also indicated a poor prognosis after transplantation. However, our results showed that there was no statistical difference in prognosis between the $M L L-\mathrm{PTD} / A B L \geq 1 \%$ and $M L L-\mathrm{PTD} / A B L<1 \%$ group based on the level of $M L L$-PTD before transplantation (All $P>0.05$, Fig. 4A-D), but the group of
$M L L-\mathrm{PTD} / A B L \geq 1 \%$ tended to have lower OS $(P=0.202$, Fig. 4B), DFS $(P=0.202$, Fig. $4 \mathrm{C})$, and have a higher $\operatorname{TRM}(P=0.105$, Fig. 4D) compared with that of $M L L-$ $\mathrm{PTD} / A B L<1 \%$ group .

\section{Factors affecting the prognosis of transplant patients with MLL-PTD}

Factors affecting the prognosis were analyzed, including transplantation age, gender, disease type, donor type, blood type compatibility (Table 3 ). There was no statistical difference in TRM $(P=0.675)$, CIR $(P=0.115)$, DFS $(P=0.151)$ and OS $(P=0.157)$ between AML and MDS-EB. Among the 12 patients who received MSDT, 2 (16.7\%) patients underwent hematological relapse both at 5 months after HSCT, and 1 patient died of pneumonia at 5.5 months. Among 36 patients who received haplo-HSCT, 4 patients (11.1\%) underwent hematological relapse at a median of 4.5 months (range, 4-9 months) after HSCT, and 8 patients (22.2\%) died due to TRM at a median of 5.3 months (range, 0.7-17.5 months). Based on the results of the analysis, it seemed that patients who 

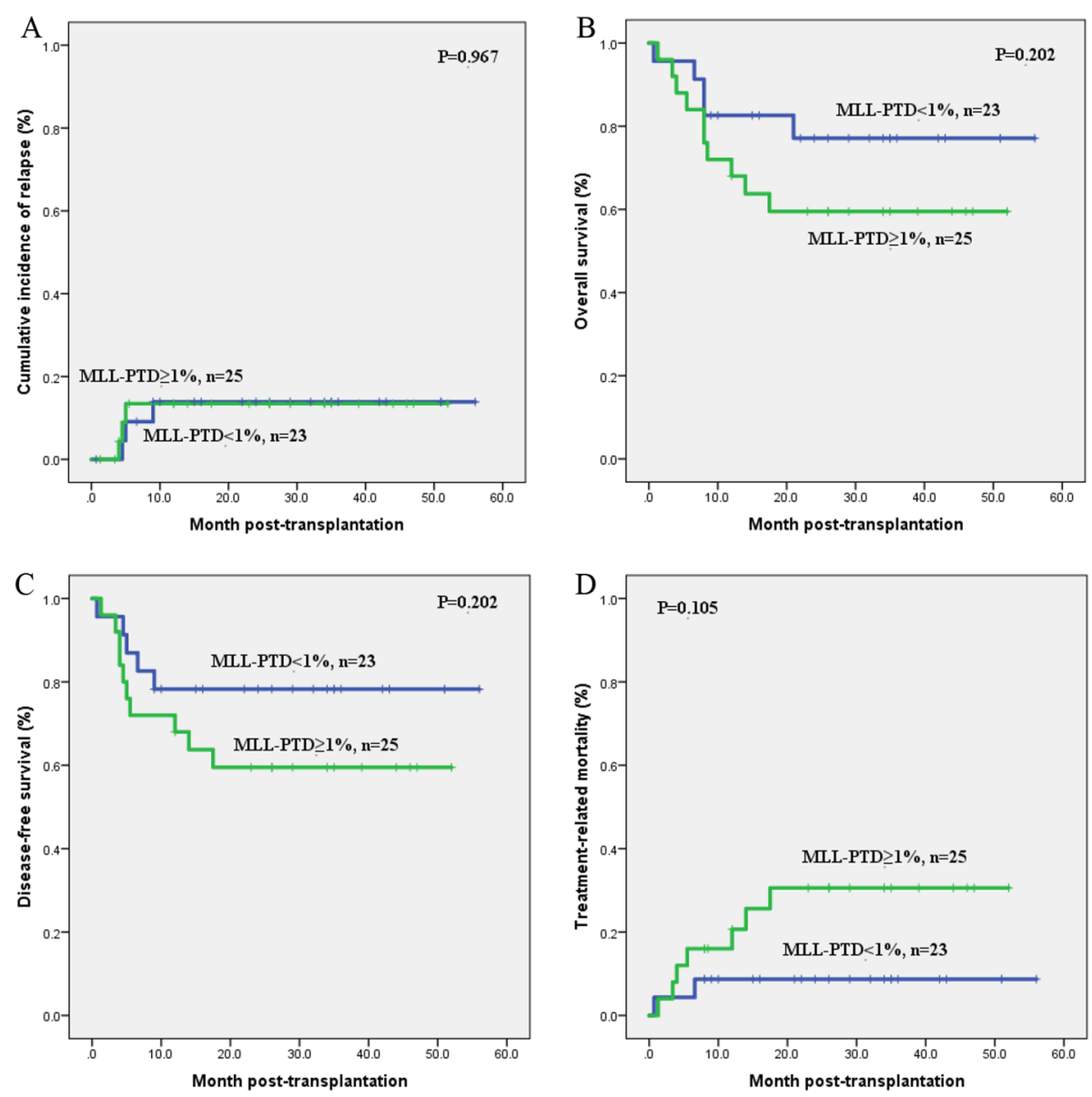

Fig. 4 Kaplan-Meier survival curves analysis of patients between MLL-PTD $<1 \%$ and MLL-PTD $\geq 1 \%$ before transplantation

received haplo-HSCT could achieve comparable outcomes compared to those who underwent MSDT (TRM: $P=0.271$; CIR: $P=0.653$; DFS: $P=0.544$; OS: $P=0.560$ ). The factor analysis of $M L L$-PTD level before and after transplantation showed that there was no statistical difference in pre-transplant $M L L$-PTD level. And posttransplant group of $M L L-\mathrm{PTD} / A B L \geq 1 \%$ had a higher CIR, a lower OS and a lower DFS than that of group of $M L L$-PTD $/ A B L<1 \%$ (all $P<0.001$ ). In addition, other factors such as age, pre-transplant FCM, WT1 status and prophylactic DLI have no significant impact on prognosis. The ABO blood type and FLT3-ITD mutation at first diagnosis were important risk factors of CIR and OS after transplantation, respectively. Incompatible $\mathrm{ABO}$ blood type indicated a higher CIR than that of compatible $A B O$ blood type, and patients with FLT3-ITD mutation had a low OS than that of without FLT3-ITD (Table 3).

\section{Comparison of MLL-PTD and other MRD parameters}

After transplantation, 8 patients were detected $M L L$ $\mathrm{PTD} / A B L \geq 1.0 \%$ at a median of 3 months. Of the 8 patients, 7 patients were simultaneously (5 patients) or subsequently ( 2 patients) MRD positive detected by FCM at a median of 4.25 months (range,3-12 months), and 6 patients ultimately progressed to hematological relapse at a median of 2 months (range, $0.25-6$ months) from the first time $M L L-\mathrm{PTD} / A B L \geq 1.0 \%$, half of whom receiving chemotherapy plus DLI. Finally, 2 patients receiving chemotherapy plus DLI became MRD negative gradually.

WT1 has been confirmed in previous studies to be an effective indicator of MRD monitoring and implementing interventions [21]. In order to analyze the specificity and sensitivity of $M L L$-PTD compared with $W T 1$, we showed in Table 2 the dynamic changes of expression of $M L L$-PTD and WT1 at the initial diagnosis and different time points before and after transplantation. All 6 relapsed patients were detected $M L L$-PTD positive prior to relapse, while only 4 patients were detected positive for WT1. As shown in Table 2, the expression levels of $M L L-\mathrm{PTD}$ and $W T 1$ both changed with the tumor burden. However, within post-transplant 3 months, MLLPTD seemed be more sensitive than WT1 for MRD 
Table 3 Univariate analysis of the variables affecting hematological TRM, CIR, DFS and OS in patients with MLL-PTD after allo-HSCT

\begin{tabular}{|c|c|c|c|c|c|}
\hline \multirow[t]{2}{*}{ Variables } & \multirow[t]{2}{*}{ Number (n,\%) } & \multicolumn{4}{|c|}{$P$ value } \\
\hline & & TRM & CIR & DFS & os \\
\hline Age of recipient & & 0.965 & 0.291 & 0.410 & 0.442 \\
\hline$<50$ years & $31(64.6 \%)$ & & & & \\
\hline$\geq 50$ years & $17(35.4 \%)$ & & & & \\
\hline Underlying disease & & 0.675 & 0.115 & 0.151 & 0.157 \\
\hline AML & $33(68.8 \%)$ & & & & \\
\hline MDS-EB1/2 & $15(31.2 \%)$ & & & & \\
\hline ABO compatibility & & 0.264 & 0.009 & 0.38 & 0.484 \\
\hline Compatible & $24(50.0 \%)$ & & & & \\
\hline Incompatible & $24(50.0 \%)$ & & & & \\
\hline Donor type & & 0.271 & 0.653 & 0.544 & 0.560 \\
\hline HLA-matched sibling & $12(25.0 \%)$ & & & & \\
\hline Haploidentical & $36(75.0 \%)$ & & & & \\
\hline Prophylactic DLI & $2(4.2 \%)$ & 0.325 & 0.591 & 0.735 & 0.702 \\
\hline FLT3-ITD positive & $10(20.8 \%)$ & 0.067 & 0.868 & 0.068 & 0.041 \\
\hline Pre-transplantation FCM & & 0.056 & 0.504 & 0.291 & 0.232 \\
\hline Negative & 23(47.9\%) & & & & \\
\hline Positive & $25(52.1 \%)$ & & & & \\
\hline Pre-transplantation WT1 & & 0.339 & 0.166 & 0.843 & 0.854 \\
\hline $\mathrm{WT} 1<0.6 \%$ & 19(40.4\%) & & & & \\
\hline WT1 $\geq 0.6 \%$ & $28(59.6 \%)$ & & & & \\
\hline Pre-transplantation MLL-PTD & & 0.105 & 0.967 & 0.202 & 0.202 \\
\hline MLL-PTD/ABL $\geq 1.0 \%$ & $25(52.1 \%)$ & & & & \\
\hline MLL-PTD/ABL $<1.0 \%$ & 23(47.9\%) & & & & \\
\hline Post-transplantation MLL-PTD & & 0.277 & $<0.001$ & $<0.001$ & $<0.001$ \\
\hline MLL-PTD/ABL $\geq 1.0 \%$ & $8(16.7 \%)$ & & & & \\
\hline MLL-PTD/ABL $<1.0 \%$ & $38(79.2 \%)$ & & & & \\
\hline
\end{tabular}

TRM treatment-associated mortality, CIR cumulative incidence of relapse, DFS disease-free survival, OS overall survival, $H L A$ human leukocyte antigen, allo- $H S C T$ allogeneic hematopoietic stem cell transplantation, $D L /$ donor lymphocyte infusion, MLL-PTD mixed lineage leukemia-partial tandem duplication, $A M L$ acute myeloid leukemia, MDS myelodysplastic syndrome

monitoring $\left(P_{+1}\right.$ month $=0.027 ; P_{+2}$ month $=0.009 ; P_{+3}$ month $=0.063)$.

\section{Discussion}

$M L L$-PTD is a special $M L L$ rearrangement gene. No report had focused on the predictive significance of peritransplant $M L L$-PTD expression on leukemia relapse after transplantation. In our retrospective study, results showed dynamic changes of $M L L$-PTD peri-transplantation, and the post-transplant $M L L-\mathrm{PTD}$ level is related to the prognosis of patients.

Previous reports have established the best threshold of $M L L$-PTD at the initial diagnosis for predicting the $C R$ or relapse in AML patients $[13,15]$. However, the AML patients with $M L L$-PTD analyzed in above reports included both non-transplanted patients and transplanted patients. Since different treatments (chemotherapy and transplantation) have a great impact on the prognosis of AML patients, they also have a certain impact on the accuracy of the $M L L$-PTD threshold for predicting relapse. Allo-HSCT is one of the curative therapies currently available for AML and MDS-EB, so it is very necessary to establish an optimal threshold of post-transplant $M L L$-PTD for relapse in transplanted AML patients. In the analysis of the post-transplant best cut-off value, we found that $M L L-\mathrm{PTD} / A B L=1 \%$ can be used as the threshold for predicting relapse. Based on this result, physicians could need to pay more attention to the occurrence of relapse for post-transplant patients with $M L L-\mathrm{PTD} / A B L \geq 1 \%$. Under this condition, it is also necessary to shorten the MRD monitoring interval, or give appropriate relapse preventive interventions in combination with the clinical condition.

A stable and reliable MRD marker whose expression level needs to vary with the tumor burden. Our data showed that $M L L$-PTD levels in relapsed patients were significantly increased before relapse. Importantly, there 
was no occurrence of $M L L$-PTD turning negative or losing before relapse, which indicated that $M L L$-PTD had a certain stability and could effectively reflect the change of tumor burden. As expected, MLL-PTD was available prior to hematological relapse, but the relapse after $M L L$-PTD positive occurred at different rates. One of the explanations may be due to the patient's combination of additional mutations such as FLT3-ITD. Previous report confirms that $M L L$-PTD positive relapses harboring an additional FLT3-ITD mutation to relapse faster than other patients with $M L L-P T D$ alone [15]. In our study, the initial diagnosis of 2 relapsed patients was accompanied by FLT3-ITD mutation. They respectively relapsed at 12 days and 35 days after post-transplant $M L L$-PTD/ $A B L \geq 1 \%$, and the relapse was significantly faster than that of other relapsed patients. These data suggested $M L L$-PTD patients with other mutations such as FLT3ITD may need to be shortened intervals of MRD monitoring after transplantation. Of course, a larger sample size or data is needed in the future to further support the above result.

The timely monitoring of MRD in the early stage after transplantation was beneficial to guide early clinical intervention to improve the prognosis of patients. Some studies have confirmed that the WT1 expression level is an independent prognostic indicator that can predict clinical outcome and combined use of WT1 and flow cytometry monitoring can promote sensitivity of predicting relapse after allo-HSCT [21, 25]. For AML and MDS lacking specific markers, we usually need to combine FCM and WT1 to evaluate MRD status. In the study, $M L L$-PTD became positive before relapse and prior to flow cytometry results. Thus, in contrast to FCM, PCR-based $M L L$-PTD detection have higher sensitivity. Our data showed that $M L L$-PTD seemed to be more sensitive than WT1 in early MRD monitoring after transplantation. Furthermore, in contrast to WT1, $M L L$-PTD is more specific for the type of $M L L-P T D$ positive AML and MDS. However, for post-transplant patients with $M L L$-PTD, in order to monitor MRD more effectively and accurately, there may not be a better way than monitoring FCM, WT1 and MLL-PTD at the same time.

AML with $M L L$-PTD is a type of leukemia with a relatively poor prognosis compared with the standard-risk AML $[13,14]$. In standard-risk AML, the post-transplant overall CIR and OS are around $15-20 \%$ and $60-70 \%$ at our institute, respectively [26, 27]. Our present results showed that the overall prognosis of post-transplant MLL-PTD patients (3-year OS: 67.8\%; 3-year CIR: 13.7\%) was similar to that of standard-risk patients. In addition, the other $M L L$ rearrangement study about the transplant-related prognosis found that allo-HSCT would have a lower relapse risk and a higher survival probability compared to the results obtained from patients with chemotherapy alone [28]. The outcomes of patients with $M L L-P T D$ are similar to the above results. The posttransplant OS in our study was significantly better than that of receiving chemotherapy alone $(3-$ year OS $<40 \%)$ in previous study [5]. These data supported that allo-HSCT could achieve good therapeutic effect in patients with $M L L$-PTD at our institute. Furthermore, haplo-HSCT could achieve the similar therapeutic effect to the MSDT in patients with $M L L$-PTD. Therefore, our institution's transplant and relapse prevention system may be effective for $M L L$-PTD patients.

\section{Conclusions}

In conclusion, $M L L$-PTD expression is a sensitive and specific MRD marker for the $M L L-P T D$ patients received allo-HSCT. $M L L$-PTD expression level higher than $1.0 \%$ suggested a high risk of hematological relapse and tended to have a worse prognosis. Furthermore, allo-HSCT could achieve good therapeutic effect in patients with $M L L$-PTD AML and MDS-EB. Of course, due to the limited number of patients with $M L L$-PTD patients, we still need to continue research to accumulate more cases to further confirm the significance of $M L L$-PTD for MRD monitoring around transplantation.

\section{Abbreviations}

AML: Acute myeloid leukemia; MDS: Myelodysplastic syndrome; Allo-HSCT: Allogeneic hematopoietic stem cell transplantation; MRD: Minimal residual disease; RQ-PCR: Real-time quantitative polymerase chain reaction; TRM:

Treatment-related mortality; CIR: Cumulative incidence of relapse; OS: Overall survival; DFS: Disease-free survival; MLL: Mixed-lineage leukemia; PTD: Partial tandem duplications; CR: Complete remission; MSDT: Matched sibling donor transplantation; NR: No remission; DLI: Donor lymphocyte infusion; BM: Bone marrow; FCM: Flow Cytometry; ANC: Absolute neutrophil count; aGVHD: Acute graft versus host disease.

\section{Acknowledgments}

Not applicable.

\section{Authors' contributions}

X.S.Z. designed the study and was responsible for whole project administration. J.K., M.-G.G. and X.-S.Z. analyzed data and wrote the manuscript, J.K. and M.-G.G. contributed equally to this work.; Y.-Z.Q., Y.W., C.-H.Y., Y.-Q.S., Y.-J.C., L.-P.X., X.-H.Z., K.-Y.L. and X.-J.H. contributed to collect samples and validate results. All authors have read and approved the manuscript.

\section{Funding}

The work was supported by the National Key Research and Development Program of China (2017YFA0104500), the National Natural Science Foundation of China (grant no. 81870137) and Innovative Research Groups of the National Natural Science Foundation of China (grant no. 81621001), Beijing Municipal Science and Technology Commission (Z181100009618032).

\section{Availability of data and materials}

The datasets used and analyzed during the current study are available from the corresponding author on reasonable request. 


\section{Declarations}

\section{Ethics approval and consent to participate}

The study followed the principles of the Helsinki Declaration and was approved by the Ethics Committee of Peking University People's Hospital. All subjects obtained informed consent and all patients or their guardians signed consent forms approved by the institutional review board.

\section{Consent for publication}

Not applicable.

\section{Competing interests}

The authors declare that they have no competing interest.

\section{Author details}

'Beijing Key Laboratory of Hematopoietic Stem Cell Transplantation, Peking University People's Hospital, Peking University Institute of Hematology, National Clinical Research Center for Hematologic Disease, No 11 Xizhimen South Street, Beijing 100044, China. ${ }^{2}$ Research Unit of Key Technique for Diagnosis and Treatments of Hematologic Malignancies, Chinese Academy of Medical Sciences, 2019RU029, Beijing, China. ${ }^{3}$ Collaborative Innovation Center of Hematology, Peking University, Beijing, China. ${ }^{4}$ Peking-Tsinghua Center for Life Sciences, Beijing 100044, China.

Received: 8 June 2021 Accepted: 23 November 2021

Published online: 03 January 2022

\section{References}

1. Walter RB, Othus M, Borthakur G, Ravandi F, Cortes JE, Pierce SA, et al. Prediction of early death after induction therapy for newly diagnosed acute myeloid leukemia with pretreatment risk scores: a novel paradigm for treatment assignment. J Clin Oncol. 2011;29:4417-23.

2. Pellagatti A, Boultwood J. The molecular pathogenesis of the myelodysplastic syndromes. Eur J Haematol. 2015;95:3-15.

3. Hosono N. Genetic abnormalities and pathophysiology of MDS. Int J Clin Oncol. 2019;24:885-92.

4. Gao MG, Ruan GR, Chang YJ, Liu YR, Qin YZ, Jiang Q, et al. The predictive value of minimal residual disease when facing the inconsistent results detected by real-time quantitative PCR and flow cytometry in NPM1-mutated acute myeloid leukemia. Ann Hematol. 2020;99:73-82.

5. Weisser M, Kern W, Schoch C, Hiddemann W, Haferlach T, Schnittger S, et al. Risk assessment by monitoring expression levels of partial tandem duplications in the MLL gene in acute myeloid leukemia during therapy. Haematologica. 2005;90:881-9.

6. Schnittger S, Weisser M, Schoch C, Hiddemann W, Haferlach T, Kern W. New score predicting for prognosis in PML-RARA+, AML1-ETO+, or CBFBMYH11 + acute myeloid leukemia based on quantification of fusion transcripts. Blood. 2003:102:2746-55

7. Guerrasio A, Pilatrino C, De Micheli D, Cilloni D, Serra A, Gottardi E, et al. Assessment of minimal residual disease (MRD) in CBFbeta/MYH1 1-positive acute myeloid leukemias by qualitative and quantitative RT-PCR amplification of fusion transcripts. Leukemia. 2002;16:1176-81.

8. Poppe B, Vandesompele J, Schoch C, Lindvall C, Mrozek K, Bloomfield $C D$, et al. Expression analyses identify MLL as a prominent target of 11 q23 amplification and support an etiologic role for MLL gain of function in myeloid malignancies. Blood. 2004;103:229-35.

9. Schichman SA, Caligiuri MA, GuY, Strout MP, Canaani E, Bloomfield CD, et al. ALL-1 partial duplication in acute leukemia. Proc Natl Acad Sci U S A. 1994;91:6236-9.

10. Caligiuri MA, Schichman SA, Strout MP, Mrózek K, Baer MR, Frankel SR, et al. Molecular rearrangement of the ALL-1 gene in acute myeloid leukemia without cytogenetic evidence of 11q23 chromosomal translocations. Cancer Res. 1994;54:370-3.

11. Nilson I, Lochner K, Siegler G, Greil J, Beck JD, Fey GH, et al. Exon/intron structure of the human ALL-1 (MLL) gene involved in translocations to chromosomal region 11q23 and acute leukaemias. Br J Haematol. 1996;93:966-72.

12. Meyer C, Hofmann J, Burmeister T, Gröger D, Park TS, Emerenciano M, et al. The MLL recombinome of acute leukemias in 2013. Leukemia. 2013;27:2165-76.
13. Kong J, Zhao XS, Qin YZ, Zhu HH, Jia JS, Jiang Q, et al. The initial level of MLL-partial tandem duplication affects the clinical outcomes in patients with acute myeloid leukemia. Leuk Lymphoma. 2018;59:967-72.

14. Choi SM, Dewar R, Burke PW, Shao L. Partial tandem duplication of KMT2A (MLL) may predict a subset of myelodysplastic syndrome with unique characteristics and poor outcome. Haematologica. 2018;103:e131-4.

15. Ommen HB, Hokland P, Haferlach T, Abildgaard L, Alpermann T, Haferlach C, et al. Relapse kinetics in acute myeloid leukaemias with MLL translocations or partial tandem duplications within the MLL gene. $\mathrm{Br}$ J Haematol. 2014;165:618-28.

16. Arber DA, Orazi A, Hasserjian R, Thiele J, Borowitz MJ, Le Beau MM, et al. The 2016 revision to the World Health Organization classification of myeloid neoplasms and acute leukemia. Blood. 2016;127:2391-405.

17. Huang XJ, Liu DH, Liu KY, Xu LP, Chen H, Han W, et al. Haploidentical hematopoietic stem cell transplantation without in vitro T-cell depletion for the treatment of hematological malignancies. Bone Marrow Transplant. 2006;38:291-7.

18. Huang X, Liu D, Liu K, Xu L, Chen H, Han W, et al. Haploidentical hematopoietic stem cell transplantation without in vitro T cell depletion for treatment of hematologic malignancies in children. Biol Blood Marrow Transplant. 2009;15(1 Suppl):91-4.

19. Chinese Society of Hematology, Chinese Medical Association. The consensus of allogeneic hematopoietic transplantation for hematological diseases in China(2016)-- post- transplant leukemia relapse. Zhonghua Xue Ye Xue Za Zhi. 2016;37:846-51.

20. Liu YR, Zhang LP, Chang Y, Cheng YF, Fu JY, Li LD, et al. Clinical significance for minimal residual disease detection by 4 color flow cytometry in adult and childhood B lineage acute lymphoblastic leukemia. Zhonghua Xue Ye Xue Za Zhi. 2006;27:302-5.

21. Zhao XS, Jin $\mathrm{S}$, Zhu HH, Xu LP, Liu DH, Chen H, et al. Wilms' tumor gene 1 expression: an independent acute leukemia prognostic indicator following allogeneic hematopoietic SCT. Bone Marrow Transplant. 2012;47:499-507.

22. Martin P, Nash R, Sanders J, Leisenring W, Anasetti C, Deeg HJ, et al. Reproducibility in retrospective grading of acute graft-versus-host disease after allogeneic marrow transplantation. Bone Marrow Transplant. 1998;21:273-9.

23. Shulman HM, Sullivan KM, Weiden PL, McDonald GB, Striker GE, Sale GE, et al. Chronic graft-versus-host syndrome in man. A longterm clinicopathologic study of 20 Seattle patients. Am J Med. 1980;69:204-17.

24. Chang YJ, Xu LP, Wang Y, Zhang XH, Chen $H$, Chen YH, et al. Controlled, randomized, open-label trial of risk-stratified corticosteroid prevention of acute graft-versus-host disease after Haploidentical transplantation. J Clin Oncol. 2016;34:1855-63.

25. Zhao XS, Yan CH, Liu DH, Xu LP, Liu YR, Liu KY, et al. Combined use of WT1 and flow cytometry monitoring can promote sensitivity of predicting relapse after allogeneic HSCT without affecting specificity. Ann Hematol. 2013;92:1111-9.

26. Zhao XS, Cao LQ, Qin YZ, Yu W, Zhang XH, Xu LP, et al. Classifying AML patients with inv(16) into high-risk and low-risk relapsed patients based on peritransplantation minimal residual disease determined by CBF $\beta$ / MYH11 gene expression. Ann Hematol. 2019;98:73-81.

27. Zhao X, Wang Z, Ruan G, Liu Y, Wang Y, Zhang X, et al. Impact of pretransplantation minimal residual disease determined by multiparameter flow cytometry on the outcome of AML patients with FLT3-ITD after allogeneic stem cell transplantation. Ann Hematol. 2018;97:967-75.

28. Wang Y, Liu QF, Qin YZ, Liu DH, Xu LP, Jiang B, et al. Improved outcome with hematopoietic stem cell transplantation in a poor prognostic subgroup of patients with mixed-lineage-leukemia-rearranged acute leukemia: results from a prospective, multi-center study. Am J Hematol. 2014;89:130-6.

\section{Publisher's Note}

Springer Nature remains neutral with regard to jurisdictional claims in published maps and institutional affiliations. 